\title{
Super greens for super hearts: a smoothie approach to increase plasma lutein.
}

\author{
Rosanna W.S. Chung \\ Division of Cardiovascular Medicine, Department of Medical and Health Sciences, Linköping University, Linköping, \\ Sweden
}

\section{Abstract}

Introduction: Coronary artery disease (CAD) is one of the major causes of death worldwide. Despite extensive treatments, many patients still exhibit low-grade systemic inflammation which increases risk of new cardiovascular events. Currently, there is no standard anti-inflammatory strategy to treat CAD.

Lutein is an antioxidant abundant in the Mediterranean diet which is known to be cardioprotective. We have previously shown that plasma lutein is inversely correlated to inflammation levels in CAD patients and that it exerts direct anti-inflammatory effects on patients' immune cells ex vivo. We have also demonstrated that consuming spinach in the form of smoothie can maximize lutein liberation in vitro. The present study is a feasibility study in preparation for a cross-over intervention study in CAD patients. It aimed to compare the efficacy and practicality of raising plasma lutein using lutein-rich smoothies or lutein supplements.

Materials and methods: 7 healthy participants were advised to consume 1) $85 \mathrm{~g}$ spinach in smoothies $\geq 5$ days/week or 2) $10 \mathrm{mg}$ lutein supplements daily in a cross-over design in two separate 8-week interventions while keeping their usual diets. A 6-week washout period during which their normal diet was maintained separates the two intervention periods. Training on smoothie making was provided. Adherence was recorded daily, and the practicality was ranked in surveys fortnightly. Plasma was collected on 11 occasions. Plasma carotenoids (lutein, $\beta$-cryptoxanthin, lycopene, $\alpha$-carotene and $\beta$-carotene) were quantified by HPLC.

Results: Both interventions received the two highest scores in terms of taste, ease of use and time consumption. Plasma lutein was increased by both interventions while no change was found in other plasma carotenoids. The lutein-raising effect was higher in supplements compared to smoothies. The smoothies raised plasma lutein levels on an average of $117 \%, 143 \%, 167 \%$ and $142 \%$ from baseline after 2, 4, 6 and 8 weeks of consumption respectively $\left(P_{\text {for trend }}=0.003\right)$. The lutein supplements increased plasma lutein on an average of $519 \%, 637 \%, 672 \%$ and $715 \%$ from baseline after $2,4,6$ and 8 weeks respectively $\left(P_{\text {for trend }}=0.002\right)$. Lutein returned to baseline levels for all participants 4 and 6 weeks after the smoothies and 8 and 10 weeks after the supplements.

Discussion: Both methods are effective in increasing plasma lutein in healthy humans. Although the supplements exert higher luteinraising effects compared to the smoothies, further testing on subjects with CAD is required to determine if the observed effects lead to inflammation reduction with clinical significance.

\section{Conflict of Interest}

There is no conflict of interest. 